BULL. AUSTRAL. MATH. SOC.

MOS $4 I A 50,4 \mid A 60$

VOL. $6(1972), 307-312$.

\title{
Functions of asymptotic expansions
}

\section{E.R. Love}

Led on by a paper of Brown and Dancer with the same title, this paper gives two further theorems on asymptotic expansions of composite functions.

In their article with the above title, Brown and Dancer extended a theorem of Entringer on asymptotic behaviour, their aim being to obtain an asymprotic expansion of a composite function from that of the inner constituent function. Since many important asymptotic expansions involve complex variables almost inherently, it seems appropriate to extend their theorem, which involves a real variable and indeed a monotonic function, to functions of a complex variable. Theorem $l$ is the outcome of this.

Nevertheless, there are asymptotic expansions of composite functions which can be obtained in a manner which is closer to the formal substitution procedure often used with convergent power series. One instance is the complex version of the example used by Brown and Dancer in [1] to illustrate their theorem: the derivation of the asymptotic expansion for $\Gamma(z+1)$ from that for $\log \Gamma(z+1)$. Theorem 2 justifies generally the formal substitution of an asymptotic expansion in a convergent power series.

THEOREM 1. Let $S=\{z: \alpha \leq \arg z \leq \beta\}$, where $-\pi \leq \alpha<\beta \leq \pi$, be a sector in the complex plane; and let $m$ and $R$ be positive constants. If

$$
|f(z)| \rightarrow \infty \text { and } g(z)=f(z)+o\left(z^{-m}\right) \text { as } z \rightarrow \infty \text { in } S \text {, }
$$

and if

Received 10 November 1971. 
$h(z)$ is regular, and $h^{\prime}(z) / h(z)$ bounded, in $|z|>R$;

then

$$
\frac{h(g(z))}{h(f(z))}=1+o\left(z^{-m}\right) \text { as } z \rightarrow \infty \text { in } S .
$$

Proof. (i) If $|w| \leq \frac{1}{2}$,

$$
\begin{aligned}
\left|\frac{w}{1 !}+\frac{w^{2}}{2 !}+\frac{w^{3}}{3 !}+\ldots\right| & \leq|w|+|w|^{2}+|w|^{3}+\ldots \\
& =\frac{|w|}{1-|w|} \leq \frac{|w|}{1-\frac{1}{2}}=2|w| ;
\end{aligned}
$$

and so

$$
|\exp w-1| \leq 2|w| \text { whenever } 2|w| \leq 1 \text {. }
$$

(ii) $h(z)$ has no zero in $|z|>R$. For otherwise Taylor's Theorem shows that $h^{\prime}(z) / h(z)$ has a simple pole there, contrary to its assumed boundedness, or else that $h(z)$ is identically zero, contrary to the implied hypothesis that $h^{\prime}(z) / h(z)$ is a function.

(iii) Let $z$ be a straight line segment, from $z_{1}$ to $z_{2}$, wholly in $|s|>R$. Then

$$
\int_{l} \frac{h^{\prime}(s)}{h(s)} d s=\log _{(2)} h\left(z_{2}\right)-\log _{(1)} h\left(z_{1}\right)
$$

where $\log _{(1)}$ and $\log _{(2)}$ are branches of the many-valued logarithm. So

$$
\begin{gathered}
\exp \left(\int_{2} \frac{h^{\prime}(s)}{h(s)} d s\right)=\frac{\exp \left(\log (2)^{\left.h\left(z_{2}\right)\right)}\right.}{\exp \left(\log (1)^{h\left(z_{1}\right)}\right.}=\frac{h\left(z_{2}\right)}{h\left(z_{1}\right)}, \\
\left|\frac{h\left(z_{2}\right)}{h\left(z_{1}\right)}-1\right|=\left|\exp \left(\int_{2} \frac{h^{\prime}(s)}{h(s)} d s\right)-1\right| \leq 2\left|\int_{l} \frac{h^{\prime}(s)}{h(s)} d s\right|
\end{gathered}
$$

by (1), provided that the last expression is at most 1 . Consequently, if $A$ is the assumed bound for $\left|h^{\prime}(s) / h(s)\right|$ in $|s|>R$,

$$
\left|\frac{h\left(z_{2}\right)}{h\left(z_{1}\right)}-1\right| \leq 2 A\left|z_{2}-z_{1}\right| \text { if } 2 A\left|z_{2}-z_{1}\right| \leq 1 \text {. }
$$

(iv) There are $\rho_{1}>0$ and $B>0$ such that, for all $z$ in $S$ 
with $|z| \geq \rho_{1}$,

$$
|f(z)|>2 R \text { and }|g(z)-f(z)|<B|z|^{-m}
$$

and there is $\rho_{2} \geq \rho_{1}$ such that $B \rho_{2}^{-m}<R$. So, for all $z$ in $S$ with $|z| \geq \rho_{2}$,

$$
|f(z)|>2 R \text { and }|g(z)-f(z)|<R \text {. }
$$

The triangle with vertices $0, f(z)$ and $g(z)$ in the Argand diagram therefore has the side joining $f(z)$ and $g(z)$ entirely outside the circle with centre 0 and radius $R$.

$$
\text { Taking } z_{1}=f(z) \text { and } z_{2}=g(z) \text { in }(2) \text {, }
$$

$$
\left|\frac{h(q(z))}{h(f(z))}-1\right| \leq 2 A|g(z)-f(z)|
$$

for all $z$ in $S$ such that $|z| \geq \rho_{2}$, provided that the right side is at most 1 . Hence, for all $z$ in $S$ such that $|z| \geq \max \left(\rho_{2},(2 A B)^{1 / m}\right)$,

$$
\left|\frac{h(g(z))}{h(f(z))}-1\right| \leq 2 A B|z|^{-m}
$$

as required.

REMARKS. The hypothesis that $h^{\prime}(z) / h(z)$ is bounded in an annular neighbourhood of $\infty$ might be thought to be more drastically restrictive here than in the real variable context of [1]. However the product and quotient of two functions $h$ satisfying this hypothesis also satisfy it. Since all linear functions satisfy it, this shows that all polynomials and rational functions satisfy it. So also do $\exp z$ and $\exp (1 / z)$; but $\exp \left(z^{2}\right)$ does not, nor indeed does any integral function of finite order exceeding 1 .

Other functions which do not satisfy this hypothesis are $\cos z$ and sinz, although only because of their behaviour on the real axis. They show that the sum and difference of functions which satisfy the hypothesis may not satisfy it, since $\exp ( \pm i z)$ do satisfy it. However the sum and difference of two asymptotic expansions present no problems, so that this feature of the hypothesis may be no handicap. 
The functions $f$ and $g$ do not need to be regular (holomorphic) for Theorem 1. So it is conceivable that their values for large $z$ may be confined to a sector $T$, even if $S$ is nearly the whole plane. In such a case the boundedness requirement on $h^{\prime}(z) / h(z)$ is only needed in $T$, as we see from (2) and (3). Consequently wider classes of functions $h$ may then be admissible.

In Theorem 2 we suppose that the function $g$ takes small values when $z$ is large, in contrast to Theorem 1 . Consequently the hypothesis on $h$ refers to a neighbourhood of 0 , instead of one of $\infty$.

THEOREM 2. Let $S=\{z: \alpha \leq \arg z \leq \beta\}$ as before. If

$$
g(z) \sim \sum_{r=1}^{\infty} \frac{a_{p}}{z^{r}} \text { as } z+\infty \text { in } S
$$

and

$$
h(w)=\sum_{n=1}^{\infty} b_{n} w^{n} \text { in a neighbourhood of } w=0 \text {, }
$$

then

$$
h(g(z)) \sim \sum_{n=1}^{\infty} \frac{c_{n}}{n} \text { as } z+\infty \text { in } s,
$$

where $c_{n}$ is the coefficient of $z^{-n}$ in the formal expansion of

$$
\sum_{n=1}^{\infty} b_{n}\left(\sum_{r=1}^{\infty} \frac{a_{r}}{r}\right)^{n}
$$

Proof. If $n$ is any positive integer, multiplication of asymptotic expansions gives

$$
g(z)^{n} \sim \sum_{r=1}^{\infty} \frac{a_{n, r}}{z^{r}} \text { as } z \rightarrow \infty \text { in } S
$$

where $a_{1, r}=a_{r}$ for each positive integer $r, a_{n, r}=0$ for $r<n$, and

$$
a_{n+1, r}=a_{1} a_{n, r-1}+a_{2} a_{n, r-2}+\ldots+a_{r-n} a_{n, n} \text { for } r>n \text {. }
$$

Next, addition of asymptotic expansions gives 


$$
\sum_{n=1}^{m-1} b_{n} g(z)^{n} \sim \sum_{r=1}^{\infty}\left(\sum_{n=1}^{m-1} b_{n} a_{n, r}\right) \frac{1}{z^{r}} \text { as } z \rightarrow \infty \text { in } S \text {, }
$$

where $m$ is any fixed positive integer. In particular,

$$
\begin{aligned}
\sum_{n=1}^{m-1} b_{n} g(z)^{n} & \left.=\sum_{r=1}^{m-1} \int_{n=1}^{m-1} b_{n} a_{n, r}\right) \frac{1}{z^{r}}+o\left(\frac{1}{z}\right) \\
& =\sum_{r=1}^{m-1}\left(\sum_{n=1}^{r} b_{n} a_{n, r}\right) \frac{1}{z^{r}}+o\left(\frac{1}{z}\right)
\end{aligned}
$$

as $z \rightarrow \infty$ in $S$. Observe that the step following (4), curtailing the inner summation, could not have been taken earlier because it is false for $r \geq m$. The definition of $c_{p}$ now gives

$$
\sum_{n=1}^{m-1} b_{n} g(z)^{n}=\sum_{r=1}^{m-1} \frac{c_{r}}{z^{r}}+o\left(\frac{1}{z}\right) \text { as } z \rightarrow \infty \text { in } S \text {. }
$$

Since

$$
g(z)=\frac{a_{1}}{z}+o\left(\frac{1}{z}\right) \text { as } z+\infty \text { in } S \text {, }
$$

there are positive constants $A$ and $B$ such that

$$
|g(z)| \leq \frac{A}{|z|} \text { for } z \text { in } S \text { with }|z|>B .
$$

Let $R$ be the radius of a $w$-neighbourhood in which $h(w)$ is expressed as the sum of its Taylor series as in the hypothesis. For $z$ in $S$ with $|z|>\max (2 A / R, B)$,

$$
\begin{aligned}
\left|\sum_{n=m}^{\infty} b_{n} g(z)^{n}\right| & \leq \sum_{n=m}^{\infty}\left|b_{n}\right|\left(\frac{A}{|z|}\right)^{n} \\
& <\left(\frac{A}{|z|}\right)^{m} \sum_{n=m}^{\infty}\left|b_{n}\right|\left(\frac{R}{2}\right)^{n-m}=\left(\frac{2 A}{R|z|}\right)^{m} \sum_{n=m}^{\infty}\left|b_{n}\right|\left(\frac{1}{2} R\right)^{n},
\end{aligned}
$$

the last series being convergent.

From (5) and (6) we now have

$$
h(g(z))=\sum_{n=1}^{m-1} b_{n} g(z)^{n}+\sum_{n=m}^{\infty} b_{n} g(z)^{n}=\sum_{r=1}^{m-1} \frac{c_{x}}{z^{r}}+o\left(\frac{1}{z^{m}}\right)
$$


as $z \rightarrow \infty$ in $S$. This holds for each fixed $m$, as required.

EXAMPLE. From the expansion $[2, p .53]$

(7) $\quad \log \Gamma(z+1)-\left(z+\frac{1}{2}\right) \log z+z-\frac{1}{2} \log (2 \pi) \sim \sum_{n=1}^{\infty} \frac{(-1)^{n-1} B}{2 n(2 n-1)} \cdot \frac{1}{z^{2 n-1}}$

as $z \rightarrow \infty$ in $|\arg z| \leq \pi-\varepsilon$, we obtain [compare 2, p. 57]

$$
\frac{\Gamma(z+1)}{(z / e)^{2} \sqrt{2 \pi z}} \sim 1+\sum_{n=1}^{\infty} \frac{c_{n}}{z^{n}}
$$

as $z+\infty$ in $|\arg z| \leq \pi-\varepsilon$, by applying Theorem 2 with $g(z)$ equal to the left side of $(7)$ and $h(w)=\exp w-1$.

\section{References}

[1] A. Brown and E.N. Dancer, "Functions of asymptotic expansions", BuzZ. Austral. Math. Soc. 4 (1971), 255-258.

[2] E.T. Copson, Asymptotic expansions (Cambridge Tracts in Mathematics and Mathematical Physics, No. 55, Cambridge University Press, Cambridge, 1965).

Department of Mathematics, University of Melbourne, Parkville, Victoria. 